\title{
MATHEMATICAL MODELLING AND COMPUTER SIMULATION OF TEMPERATURE DISTRIBUTION IN INHOMOGENEOUS COMPOSITE SYSTEMS WITH IMPERFECT INTERFACE
}

\author{
Pratibha JOSHI ${ }^{\mathrm{a}}$, Manoj KUMAR ${ }^{\mathrm{b}}$ \\ ${ }^{a}$ Department of Mathematics, College of Engineering Studies, University of Petroleum and Energy \\ Studies, Energy Acres, VPO-Bidholi, PO-Prem Nagar, 248007 Dehradun, Uttarakhand, India \\ ${ }^{b}$ Department of Mathematics, Motilal Nehru National Institute of Technology, \\ Allahabad-211004 (Uttar Pradesh) India
}

Received 07 January 2014; accepted 27 September 2014

\begin{abstract}
Many studies have been done previously on temperature distribution in inhomogeneous composite systems with perfect interface, having no discontinuities along it. In this paper we have determined steady state temperature distribution in two inhomogeneous composite systems with imperfect interface, having discontinuities in temperature and heat flux using decomposed immersed interface method and performed the numerical simulation on MATLAB.
\end{abstract}

Keywords: elliptic interface problems, steady state heat conduction, immersed interface method, composite systems.

\section{Introduction}

Studies regarding steady state temperature distribution in composite structures have become of great importance due to expanded use of composite material in many industrial applications e.g. aerospace, automotive, shipbuilding, nuclear and many others. We need thermal profiles of modern composite materials in various engineering problems e.g. construction of furnaces, conductors (Seyidmamedov, Ozbilge 2006) thermal protection applications, laminated and fiber reinforced materials, cooling problems etc. In many modern composite systems e.g. thermal barrier coatings, optical coatings, electronic packages, we rely on integrity of interfaces for material reliability. Hence, we investigate their thermal performance by a qualitative thermal analysis.

The phenomenon of steady state heat conduction in composite structures, Joshi and Kumar (2012) is governed by elliptic boundary value problems with in- terfaces, which are often called elliptic interface problems. The difficulty to solve such problems is caused when there is discontinuity across the interfaces. Discontinuity can be either in the solution or flux or the coefficient in the governing partial differential equations. Standard finite difference methods may not give accurate results for these problems especially near the interfaces because of the discontinuity.

Previously a number of approaches have been proposed for solving problems of steady state heat conduction in different types of composite systems. Most of them are capable of solving those problems only in which there is no discontinuity along the interface i.e. the contact between two materials is perfect.

In Seyidmamedov and Ozbilge (2006), a numerical scheme is used to solve transmission problems that arise in steady state heat conduction. Two types of problems; the two layered nonhomogeneous medium interface problems and three-layered medium 
(conductor-isolator-conductor) interface problems are modelled and solved by a conservative finite difference scheme on non uniform mesh. In Noor and Burton (1991), a predictor-corrector method is proposed for determining temperature and heat flux in composite plates and shells. The method is based on using the information obtained from a simple two-dimensional shear deformation theory to correct certain key elements of the computational model and hence improve the response predictions. The plates are assumed to be antisymmetrically laminated with respect to the middle plane and the cylinders are constructed of orthotropic layers.

In Berger, Skilowitz and Tewary (2000), Green's functions are obtained through a Fourier representation for solving steady state heat conduction problems in anisotropic bimaterials. The Green's function is shown to degenerate to the usual logarithmic potential for steady state heat conduction in both uniform anisotropic and isotropic solids. To obtain the regiondependent parts of the Green's function, the homogeneous solution is written using the virtual force method. In Datskovskii and Yakunin (2005), steady state heat conduction problems in a composite region with boundary conditions of the fourth kind are solved by a Fourier method. Solution is constructed in each individual region.

In Zarubin and Radikov (2007), temperature state of an inhomogeneous body has been determined by a numerical method based on employing a dual variational formulation of the problem; which includes alternative functionals, where the direct functional reaches a minimal value and the alternative functional a maximal value, which allows finding the meansquare error of the solution.

A fundamental heat conduction problem has been solved by Ma and Chang (2004) in anisotropic multilayered media having steady state heat temperature and heat flux fields in each layer subjected to prescribed temperature on the surface and perfect thermal contact. The anisotropic multi-layered heat conduction problem is reduced to equivalent isotropic ones by a linear coordinate transformation without complicating the geometry and boundary conditions of the problem and its analytical exact solution has been determined by Fourier transform and the series expansion technique. In Xia and Jacobi (2004), the temperature distribution in a two-dimensional composite fin comprised of a fin and coating material is analyzed. An exact solution of this problem is obtained by the separation of variables.
Green's functions in boundary element approach are used to solve heat conduction problems with imperfect interface by Ang (2006) and Ang, Choo and Fan (2004). Green's functions are derived which satisfies the appropriate interface condition and solution of the governing partial differential equations is obtained which is expressed in terms of an integral taken over only the exterior boundary of the region. A particular type of jump condition has been taken where the temperature jump is proportional to the heat flux jump.

In Sheikh, Beck and Agonafer (2003), steady state heat conduction in two-layer bodies with boundary conditions of the first, second, and third kind have been studied. This study includes tables to assist the selection of eigenfunctions and computation of the eigenvalues. The computations include the contribution of contact resistance to the temperature solution. The eigenvalues are evaluated by an efficient computational approach.

In real world applications there is no perfect contact between composite systems practically. We can find different types of discontinuities in microscopic observation of such systems. In this paper decomposed immersed interface method has been applied to solve problems with imperfect contact and a general class of discontinuous jump conditions in temperature and flux. A decomposed immersed interface method is presented by Berthelsen (2004) where a correction term is introduced to standard difference stencil on the right hand side only so that the resulting linear system remains symmetric and diagonally dominant which can be solved by standard solvers. This correction term is decomposed in both cartesian directions. We have applied this method in inhomogeneous composite systems and computational results are discussed in graphical and tabular form. Our motivation towards this work is the industrial importance of steady state heat conduction in composite systems (Kumar, Joshi 2012a, 2013) and the efficiency and robustness of this method for handling interfaces. The proposed method is second order accurate, fast, efficient and easy to apply.

The remainder of the paper is constructed as follows: In Section 1, we have explained the decomposed immersed interface method. In Section 2, two steady state heat conduction problems in inhomogeneous composite systems with discontinuities along the interface have been solved and their computer simulations have been performed and in the last Section, we have summarized our work with some conclusions. 


\section{A decomposed immersed interface method for elliptic interface problems}

The original immersed interface method (IIM) given by Li (1994), solves any elliptic interface problem by including the interfacial boundary conditions into the finite difference discretization in such a way that it preserves the jumps in the solution and its derivatives. The original IIM adds additional nodes to numerical stencil, leading to a non-symmetric matrix. This nonsymmetric matrix reduces the numbers of efficient numerical solvers to be used and convergence is not always guaranteed (Kumar, Joshi 2012b). This increases the computational cost of solving this matrix.

A decomposed immersed interface method is introduced by Berthelsen (2004) which keeps the standard finite difference stencil making only corrections to the right hand side of the problem. As a result, the linear system remains symmetric and diagonally dominant. The order of accuracy is improved by including more jump conditions. In this method, the interface is represented by a level set function.

We find this method appropriate for solving the class of problems discussed in section 1 because we have to add correction term only for irregular points. At regular points jumps becomes zero the descretization reduces to the standard finite difference scheme. The method is second order accurate and easy to implement. Let us discuss the method in detail.

Suppose we have a domain $\Omega$ divided into two (or more) subdomains $\Omega^{+}$and $\Omega^{-}$by a lower dimensional interface $\Gamma$. Let us consider an elliptic boundary value problem

$$
\frac{\partial}{\partial x}\left(k \frac{\partial u}{\partial x}\right)+\frac{\partial}{\partial y}\left(k \frac{\partial u}{\partial y}\right)=f(x, y), \quad x \in \Omega
$$

with Dirichlet boundary conditions

$$
u(x, y)=g(x, y), \quad(x, y) \in \delta \Omega,
$$

where $\delta \Omega$ is the boundary of the domain $\Omega$. Suppose $k$ and source term $f$ have jumps across the interface $\Gamma$, i.e.

and

$$
k= \begin{cases}k^{+}, & (x, y) \in \Omega^{+} \\ k^{-}, & (x, y) \in \Omega^{-}\end{cases}
$$

$$
f= \begin{cases}f^{+}, & (x, y) \in \Omega^{+} \\ f^{-}, & (x, y) \in \Omega^{-}\end{cases}
$$

which can cause discontinuities in the solution and its derivatives specified as

$$
\begin{aligned}
& {[u]=w(x, y), \quad(x, y) \in \Gamma} \\
& {\left[k u_{n}\right]=v(x, y), \quad(x, y) \in \Gamma,}
\end{aligned}
$$

where $\left[u_{n}\right]=\frac{\partial u}{\partial n}=\nabla u \cdot \vec{n}$ is normal derivative of $u, \vec{n}$ is the local unit normal vector to the interface pointing towards the $\Omega^{+}$- region and the jumps are defined as the limiting values

$$
\begin{aligned}
& {[u]=\lim _{(x, y) \rightarrow \Gamma^{+}} u(x, y)-\lim _{(x, y) \rightarrow \Gamma^{-}} u(x, y)=u^{+}-u^{-}} \\
& {\left[k u_{n}\right]=\lim _{(x, y) \rightarrow \Gamma^{+}} k(x, y) u_{n}(x, y)-} \\
& \lim _{(x, y) \rightarrow \Gamma^{-}} k(x, y) u_{n}(x, y)=k^{+} u_{n}{ }^{+}-k^{-} u_{n}{ }^{-} .
\end{aligned}
$$

Level set representation of the interface. Let us define a smooth auxiliary function $\phi(x, y)$ as

$$
\phi(x, y)= \pm d,
$$

where $d$ is the shortest distance to the interface. The interface is represented by the zero level set of the function $\phi$,

$$
\Gamma=\left\{(x, y) \in R^{2} \mid \phi(x, y)=0\right\} .
$$

The sign of $\phi$ indicates whether $(x, y)$ is in the $\Omega^{+}-$region (in case of positive sign) or in the $\Omega^{-}-$ region (in case of negative sign) i.e.

$$
\Omega=\left\{\begin{array}{ll}
\Omega^{-}, & \phi<0 \\
\Omega^{+}, & \phi \geq 0
\end{array} .\right.
$$

Numerical discretization. Suppose we have a rectangular domain $\Omega=[a, b] \times[c, d]$. The uniform grid is defined as

$$
x_{i}=a+i h, y_{j}=c+j h,
$$

where $h=\frac{b-a}{M}=\frac{d-c}{N}$ and $0 \leq i \leq M, 0 \leq j \leq N$.

A grid point is called regular if the neighbouring nodes are on the same side of the interface whereas a grid point is irregular if at least one neighbouring node is on the other side of the interface. Now elliptic equation (1) can be approximated by the following discretization

$$
\begin{aligned}
& \frac{k_{i+1 / 2, j}\left(U_{i+1, j}-U_{i, j}\right)-k_{i-1 / 2, j}\left(U_{i, j}-U_{i-1, j}\right)}{h^{2}}+ \\
& \frac{k_{i, j+1 / 2}\left(U_{i, j+1}-U_{i, j}\right)-k_{i, j-1 / 2}\left(U_{i, j}-U_{i, j-1}\right)}{h^{2}}=f_{i, j},
\end{aligned}
$$

where $U_{i, j}=u\left(x_{i}, y_{j}\right), f_{i, j}=f\left(x_{i}, y_{j}\right)$ and $k_{i+1 / 2, j}=k\left(x_{i}+h / 2, y_{j}\right)$.

A correction term $C_{i, j}$ is introduced on the right hand side of equation (5) to make the numerical discretization well defined at irregular nodes and should vanish at regular nodes. This correction term can be decomposed directionwise since jump condition can 
be decomposed in the $\mathrm{x}$ - and $\mathrm{y}$ - directions allowing for a dimension by dimension approach. Hence we can define correction term as

$$
C_{i, j}=C_{i, j}^{x}+C_{i, j}^{y} .
$$

If we will evaluate correction term in $\mathrm{x}$ direction we can get another correction term by the similar procedure in y-direction. Hence we will discuss the procedure of evaluating correction term in only $\mathrm{x}$-direction.

Let us suppose that interface is located at $x_{\Gamma}=x_{i}+\sigma h, 0 \leq \sigma \leq 1, \sigma=\frac{\phi_{i}}{\phi_{i}-\phi_{i+1}}$, where $x_{i}$ is an irregular node and $\phi_{i}=\phi\left(x_{i}\right)$. The correction will be done in two steps. First we will correct the numerical discretization of $u_{x}$, then, the approximation of $\left(k u_{x}\right)_{x}$. The first derivative is estimated at the centre between $x_{i}$ and $x_{i+1}$. The correction of this approximation depends on what side of $x_{i+1 / 2}$ the interface is located.

Writing expression for $u\left(x_{i+1}\right)$ at $x_{\Gamma}=x_{i}+\sigma h$ for the case $\phi_{i}<0$ and $1 / 2<\sigma \leq 1$ using Taylor's series expansion

$u\left(x_{i+1}\right)=u\left(x_{\Gamma}+(1-\sigma) h\right)=u^{+}+u_{x}^{+}(1-\sigma) h+$

$\frac{1}{2} u_{x x}^{+}(1-\sigma)^{2} h^{2}+\mathrm{O}\left(h^{3}\right)=$

$u^{-}+u_{x}^{-}(1-\sigma) h+\frac{1}{2} u_{x x}^{-}(1-\sigma)^{2} h^{2}+C_{1}(x, \sigma)+\mathrm{O}\left(h^{3}\right)=$

$u\left(x_{i+1 / 2}\right)+u_{x}\left(x_{i+1 / 2}\right)(\sigma-1 / 2) h+$

$\frac{1}{2} u_{x x}\left(x_{i+1 / 2}\right)(\sigma-1 / 2)^{2} h^{2}+u_{x}\left(x_{i+1 / 2}\right)(1-\sigma) h+$

$\frac{1}{2} u_{x x}\left(x_{i+1 / 2}\right)(2 \sigma-1)(1-\sigma) h^{2}+\frac{1}{2} u_{x x}\left(x_{i+1 / 2}\right)(1-\sigma)^{2} h^{2}+$ $C_{1}(x, \sigma)+\mathrm{O}\left(h^{3}\right)=u\left(x_{i+1 / 2}\right)+u_{x}\left(x_{i+1 / 2}\right) \frac{h}{2}+$

$\frac{1}{2} u_{x x}\left(x_{i+1 / 2}\right)\left(\frac{h}{2}\right)^{2}+C_{1}(x, \sigma)+O\left(h^{3}\right)$.

Expanding $u\left(x_{i}\right)$ at $x_{i+1 / 2}$ we get $u\left(x_{i}\right)=u\left(x_{i+1 / 2}\right)-u_{x}\left(x_{i+1 / 2}\right) \frac{h}{2}+\frac{1}{2} u_{x x}\left(x_{i+1 / 2}\right)\left(\frac{h}{2}\right)^{2}+O\left(h^{3}\right)$.

From (7) we can evaluate the correction term as $C_{1}(x, \sigma)=[u]+\left[u_{x}\right](1-\sigma) h+\frac{1}{2}\left[u_{x x}\right](1-\sigma)^{2} h^{2}$.

Subtracting (8) from (7) we get

$u_{x}\left(x_{i+1 / 2}\right)=\frac{u\left(x_{i+1}\right)-u\left(x_{i}\right)}{h}-\frac{C_{1}(x, \sigma)}{h}+O\left(h^{2}\right)$.

For the case $0<\sigma \leq 1 / 2$ expanding $u\left(x_{i}\right)$ at $x_{\Gamma}=x_{i}+\sigma h$ we get

$u\left(x_{i}\right)=u\left(x_{\Gamma}-\sigma h\right)=u^{-}-u_{x}^{-} \sigma h+\frac{1}{2} u_{x x}^{-} \sigma^{2} h^{2}+O\left(h^{3}\right)=$ $u^{+}-u_{x}^{+} \sigma h+\frac{1}{2} u_{x x}^{+} \sigma^{2} h^{2}-C_{1}(x, \sigma)+O\left(h^{3}\right)=$ $u\left(x_{i+1 / 2}\right)-u_{x}\left(x_{i+1 / 2}\right)\left(\frac{1}{2}-\sigma\right) h+\frac{1}{2} u_{x x}\left(x_{i+1 / 2}\right)\left(\frac{1}{2}-\sigma\right)^{2} h^{2}-$

$u_{x}\left(x_{i+1 / 2}\right) \sigma h+\frac{1}{2} u_{x x}\left(x_{i+1 / 2}\right)(1-2 \sigma) \sigma h^{2}+$

$\frac{1}{2} u_{x x}\left(x_{i+1 / 2}\right) \sigma^{2} h^{2}-C_{1}(x, \sigma)+O\left(h^{3}\right)=u\left(x_{i+1 / 2}\right)-$

$u_{x}\left(x_{i+1 / 2}\right) \frac{h}{2}+\frac{1}{2} u_{x x}\left(x_{i+1 / 2}\right)\left(\frac{h}{2}\right)^{2}-C_{1}(x, \sigma)+O\left(h^{3}\right)$

and Expanding $u\left(x_{i+1}\right)$ at $x_{i+1 / 2}$ by Taylor's series expansion

$$
\begin{aligned}
& u\left(x_{i+1}\right)=u\left(x_{i+1 / 2}\right)+u_{x}\left(x_{i+1 / 2}\right) \frac{h}{2}+ \\
& \frac{1}{2} u_{x x}\left(x_{i+1 / 2}\right)\left(\frac{h}{2}\right)^{2}+O\left(h^{3}\right) .
\end{aligned}
$$

In this case correction term can be evaluated as

$$
C_{1}(x, \sigma)=[u]-\left[u_{x}\right] \sigma h+\frac{1}{2}\left[u_{x x}\right] \sigma^{2} h^{2} .
$$

Subtracting (9) from (10) we get

$u_{x}\left(x_{i+1 / 2}\right)=\frac{u\left(x_{i+1}\right)-u\left(x_{i}\right)}{h}-\frac{C_{1}(x, \sigma)}{h}+O\left(h^{2}\right)$.

Also, the approximation of flux $k u_{x}$ needs to be corrected, if it is discontinuous and $0<\sigma \leq 1 / 2$. Hence expanding $k u_{x}$ at $x_{i+1 / 2}$ :

$$
\begin{aligned}
& k u_{x}\left(x_{i+1 / 2}\right)=k u_{x}\left(x_{\Gamma}+\left(\frac{1}{2}-\sigma\right) h\right)=\left(k u_{x}\right)^{+}+ \\
& \left(k u_{x}\right)_{x}^{+}\left(\frac{1}{2}-\sigma\right) h+O\left(h^{2}\right)= \\
& \left(k u_{x}\right)^{-}+\left(k u_{x}\right)_{x}^{-}\left(\frac{1}{2}-\sigma\right) h+C_{2}(x, \sigma)+O\left(h^{2}\right)= \\
& k u_{x}\left(x_{i}\right)+\left(k u_{x}\right)_{x}\left(x_{i}\right) \sigma h+\left(k u_{x}\right)_{x}\left(x_{i}\right)\left(\frac{1}{2}-\sigma\right) h+ \\
& C_{2}(x, \sigma)+O\left(h^{2}\right)=k u_{x}\left(x_{i}\right)+\left(k u_{x}\right)_{x}\left(x_{i}\right) \frac{h}{2}+ \\
& C_{2}(x, \sigma)+O\left(h^{2}\right) .
\end{aligned}
$$

Similarly expanding $k u_{x}$ at $x_{i-1 / 2}$ :

$k u_{x}\left(x_{i-1 / 2}\right)==k u_{x}\left(x_{i}\right)-\left(k u_{x}\right)_{x}\left(x_{i}\right) \frac{h}{2}+O\left(h^{2}\right)$.

Hence the correction term $C_{2}$ can be evaluated from (11) as

$$
C_{2}(x, \sigma)=\left[k u_{x}\right]+\frac{1}{2}\left[\left(k u_{x}\right)_{x}\right](1-2 \sigma) h .
$$

Subtracting (13) from (12) we get

$$
\begin{aligned}
& \left(k u_{x}\right)_{x}\left(x_{i}\right)=\frac{k u_{x}\left(x_{i+1 / 2}\right)-k u_{x}\left(x_{i+1 / 2}\right)}{h}- \\
& \frac{C_{2}(x, \sigma)}{h}+O(h) .
\end{aligned}
$$


Hence for $x_{i-1} \leq x_{\Gamma} \leq x_{i+1}$, we can numerically approximate $\left(k u_{x}\right)_{x}\left(x_{i}\right)$ as

$\left(k u_{x}\right)_{x}\left(x_{i}\right)=\frac{k_{i+1 / 2, j}\left(U_{i+1, j}-U_{i, j}\right)-k_{i-1 / 2, j}\left(U_{i, j}-U_{i-1, j}\right)}{h^{2}}+$

$C_{i}+O(h)$,

with correction term

$$
C_{i}=S_{\phi} \bar{k} \frac{C_{1}(x, \sigma)}{h^{2}}+S_{\phi} \frac{C_{2}(x, \sigma)}{h},
$$

where

$$
S_{\phi}=\left\{\begin{array}{cc}
-1 & \phi_{i}<0 \\
1 & \phi_{i} \geq 0
\end{array}\right.
$$

and

$$
\begin{aligned}
& C_{1}(x, \sigma)= \begin{cases}{[u]-\lambda\left[u_{x}\right] \sigma h+1 / 2 \sigma^{2} h^{2}\left[u_{x x}\right]} & \text { if }\left(\phi_{i} \geq 0 \text { and } 0 \leq \sigma<1 / 2\right) \\
& \text { or }\left(\phi_{i}<0 \text { and } 0<\sigma \leq 1 / 2\right) \\
{[u]+\lambda\left[u_{x}\right](1-\sigma) h+1 / 2(1-\sigma)^{2} h^{2}\left[u_{x x}\right]} & \text { if }\left(\phi_{i} \geq 0 \text { and } 1 / 2<\sigma \leq 1\right) \\
\text { or }\left(\phi_{i}<0 \text { and } 1 / 2 \leq \sigma<1\right)\end{cases} \\
& C_{2}(x, \sigma)=\left\{\begin{array}{cc}
\lambda\left[k u_{x}\right]+1 / 2\left[\left(k u_{x}\right)_{x}\right](1-2 \sigma) h & \text { if }\left(\phi_{i} \geq 0 \text { and } 0 \leq \sigma<1 / 2\right) \\
0 & \text { or }\left(\phi_{i}<0 \text { and } 0<\sigma \leq 1 / 2\right) \\
0 & \text { otherwise }
\end{array}\right.
\end{aligned}
$$

where the parameters $\lambda, \sigma$ and $\bar{k}$ are defined as

$$
\begin{aligned}
& - \text { If } x_{i} \leq x_{\Gamma} \leq x_{i+1} \\
& \lambda=1, \sigma=\frac{\phi_{i}}{\phi_{i}-\phi_{i+1}} \text { and } \bar{k}=k_{i+1 / 2} ; \\
& - \text { If } x_{i-1} \leq x_{\Gamma} \leq x_{i} ; \\
& \lambda=-1, \quad \sigma=\frac{\phi_{i}}{\phi_{i}-\phi_{i-1}} \text { and } \bar{k}=k_{i-1 / 2} .
\end{aligned}
$$

The jumps become zero where solution is smooth and continuous and therefore correction term gets vanished. In that case (16) reduces to standard finite difference approximation for $\left(k u_{x}\right)_{x}$. In Berthelsen (2004) the method for approximating jump conditions is also given if they are unknown. Since the correction term is added only at right hand side, the system of linear discretized equations is a symmetric and diagonally dominant matrix and can be solved with any standard solver. In higher dimensional problem we can similarly evaluate expression for correction terms in each direction. We found this method appropriate for solving such problems because of the following reasons:

- The method is easy to implement since the correction term only needs to be added to the right hand side of the system.

- The method is second order accurate.

- The method is robust and can be applied to variety of problems.
- The coefficient matrix can be solved any standard solver.

In the next section, we have determined temperature distribution in two inhomogeneous composite systems with different types of boundary condition and discontinuous jump conditions using decomposed immersed interface method and performed their computer simulation in MATLAB 7.0.1 with computer configuration Intel Atom processor and 2 GB RAM.

\section{Computer simulation}

Problem 1. In first problem we have taken the same composite system as discussed in Datskovskii and Yakunin (2005) but with different assumptions. Hence, the boundary conditions and jump conditions are different. In Datskovskii and Yakunin (2005) the contact has been taken perfect but we assume that temperature and heat flux are discontinuous along the interface.

Mathematical model of problem 1 . We take a composite system (see Fig. 1) having two regions $R_{1}$ and $R_{2}$ with different thermal conductivity $K_{2}^{1}$ and $K_{2}^{2}$ along $y$-direction, and same thermal conductivity $K_{1}$ along $x$-direction having contact at $y=b_{1}$. We suppose temperature of each region is $T_{i}, i=1,2$. Hence according to the assumption, we have taken; steady state heat conduction in this system is modelled as following:

The governing elliptic equation is

$$
\frac{\partial}{\partial x}\left(K_{1} \frac{\partial T_{i}}{\partial x}\right)+\frac{\partial}{\partial y}\left(K_{2}^{i} \frac{\partial T_{i}}{\partial y}\right)=0, \quad i=1,2,
$$

along with boundary conditions

$$
\begin{aligned}
& \left.T_{1}\right|_{x \in\left(0, a_{1}\right), y=0}=\left.T_{2}\right|_{x \in\left(a_{1}, a_{2}\right), y=b_{1}}=T_{0}, \\
& \left.\frac{\partial T_{1}}{\partial x}\right|_{x=0, y \in\left(0, b_{1}\right)}=0, \\
& \left.\frac{\partial T_{1}}{\partial x}\right|_{x=a_{1}, y \in\left(0, b_{1}\right)}=0, \\
& \left.\frac{\partial T_{2}}{\partial x}\right|_{x=0, y \in\left(b_{1}, b_{2}\right)}=0 \\
& \left.\frac{\partial T_{2}}{\partial x}\right|_{x=a_{2}, y \in\left(b_{1}, b_{2}\right)}=0 \\
& \left.T_{2}\right|_{x \in\left(0, a_{2}\right), y=b_{2}}=T_{p}
\end{aligned}
$$

and jump conditions

$$
T_{1}-\left.T_{2}\right|_{x \in\left(0, a_{1}\right), y=b_{1}}=A,
$$




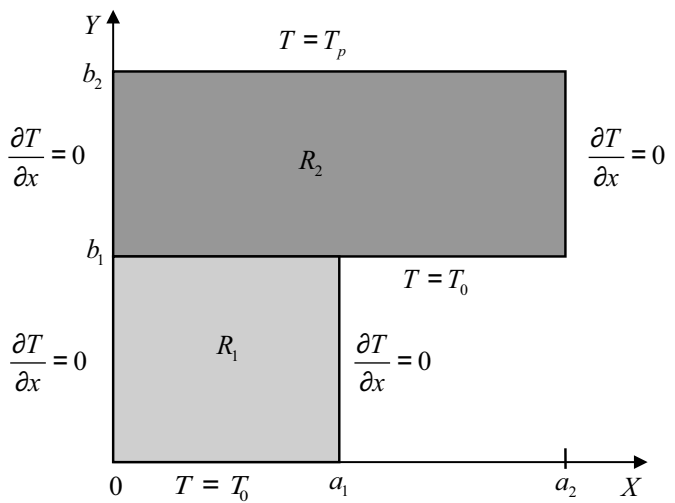

Fig. 1. Composite system with two regions

$$
\begin{aligned}
& \frac{\partial T_{2}}{\partial y}-\left.\frac{\partial T_{1}}{\partial y}\right|_{x \in\left(0, a_{1}\right), y=b_{1}}=B, \\
& K_{2}^{2} \frac{\partial T_{2}}{\partial y}-\left.K_{2}^{1} \frac{\partial T_{1}}{\partial y}\right|_{x \in\left(0, a_{1}\right), y=b_{1}}=C .
\end{aligned}
$$

We have solved the above problem using decomposed immersed interface method in MATLAB 7.0.1 taking uniform mesh. The computation has been performed for these values of parameters:

$K_{1}=50 \mathrm{~W} / \mathrm{m} \cdot \operatorname{deg}, K_{2}^{1}=100 \mathrm{~W} / \mathrm{m} \cdot \operatorname{deg}$,

$K_{2}^{2}=200 \mathrm{~W} / \mathrm{m} . \operatorname{deg}, a_{1}=0.01 \mathrm{~m}, a_{2}=0.02 \mathrm{~m}$,

$b_{1}=0.002 \mathrm{~m}, b_{2}=0.004 \mathrm{~m}, T_{0}=20 \mathrm{deg}, T_{p}=30 \mathrm{deg}$,

$A=-6 \mathrm{deg}, B=2000 \mathrm{deg} / \mathrm{m}, C=7 \times 10^{5} \mathrm{deg} / \mathrm{m}^{2}$.

The analytic solution of this problem is

$$
T_{A}(x, y)=\left\{\begin{array}{ll}
3000 y+20 & (x, y) \in R_{1} \\
5000 y+10 & (x, y) \in R_{2}
\end{array}\right. \text {. }
$$

Temperature distributions in region $\left(R_{1}\right)$ and region $\left(R_{2}\right)$ are shown in Figure 2 and Figure 3 respectively. It can be be visualized from the figures that the temperature in this system varies along height only. The figures are plotted taking $20 \times 20$ mesh. For analyzing efficiency of our approach, we have also compared the approximate numerical solution with analytical solution and demonstrated the maximum absolute error in Table 1 at some grids.

Table 1. Maximum absolute error

\begin{tabular}{|c|c|}
\hline Grids & $\left\|e_{n}\right\|_{\infty}$ by Decomposed IIM \\
\hline $10 \times 10$ & $3.197442310920451 \mathrm{e}-014$ \\
\hline $14 \times 14$ & $2.842170943040401 \mathrm{e}-014$ \\
\hline
\end{tabular}

Problem 2. We take another composite system, described in Zarubin and Radikov (2007) with different assumptions. It is a recurring element of a circular cylindrical shell of an engine combustor shown in
Figure 4. The shell consists of an outer wall of inside radius $R$ and thickness $h_{2}$ and an inner wall in which channels of rectangular cross section of width $2 b$ are milled in parallel with shell axis so that longitudinal ribs of height $L$ and width $2 \delta$ are formed between the channels. The inner wall thickness after milling is $h_{1}$.

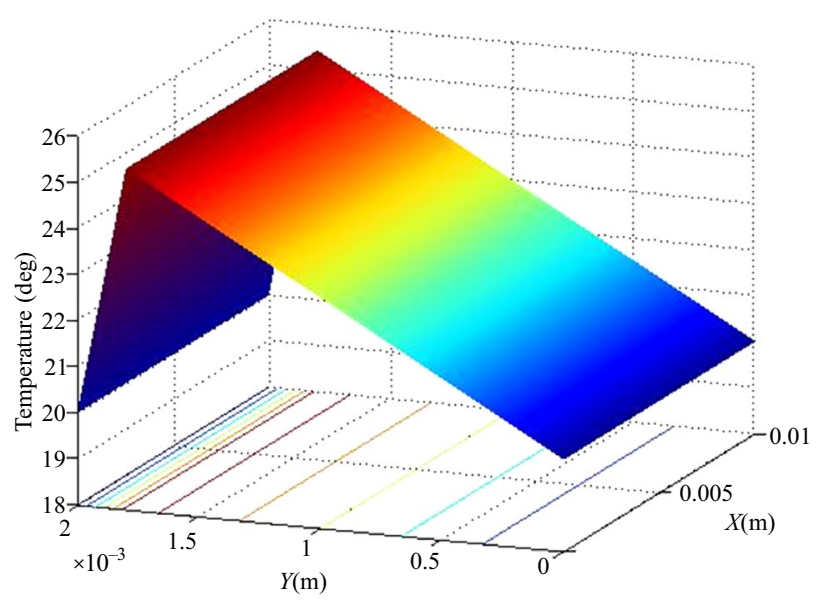

Fig. 2. Temperature distribution in first region $\left(R_{1}\right)$ in problem 1

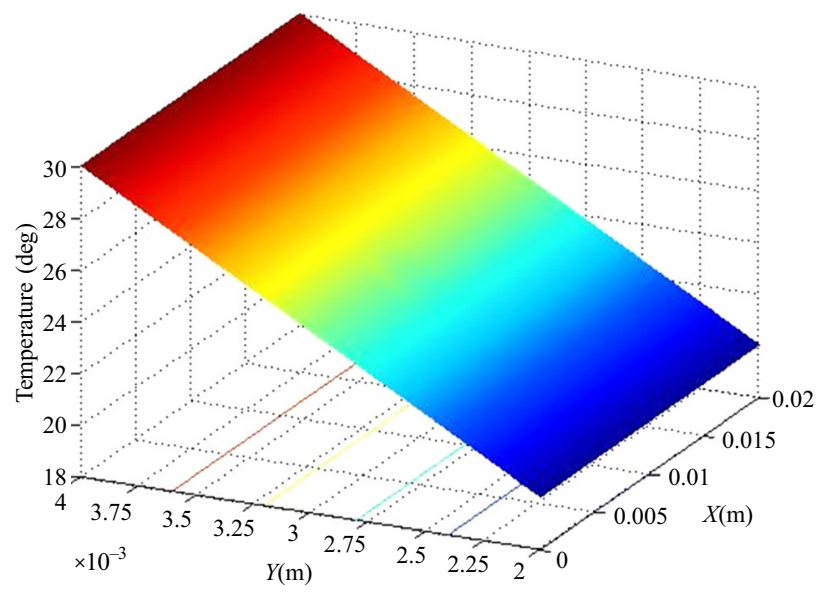

Fig. 3. Temperature distribution in second region $\left(R_{2}\right)$ in problem 1

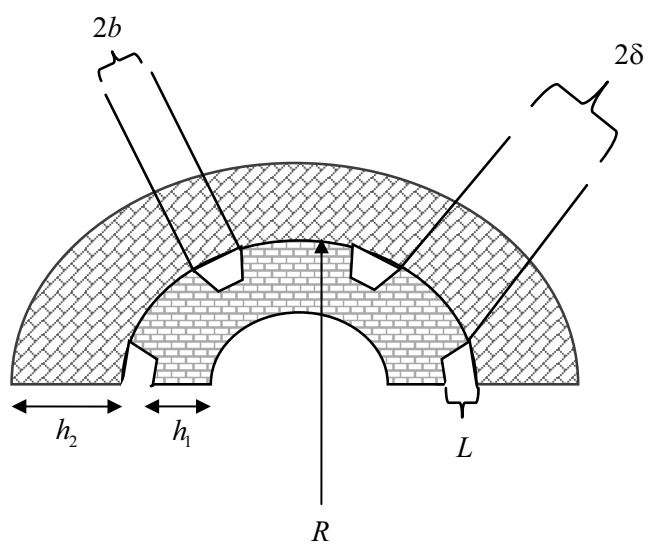

Fig. 4. A cross section of the shell of an engine combustor 
The ratio of the shell radius to thickness is much greater than unity (Balabukh et al. 1969); therefore we can represent this structure as a flat wall with straight ribs of height $L$ and width $2 \delta$ milled in this wall and spaced at intervals of $2 b$. In Zarubin and Radikov (2007), the thermal contact between the ribs and outer wall is taken to be ideal but we have assumed it to be imperfect having discontinuities in temperature as well as in heat flux along the interface. Since the operating time of engines, in which such combustor structures are employed, is relatively short; we can solve a simpler steady state heat conduction which is the limiting case of unsteady-state problem.

Mathematical model of problem 2. The recurring element of the structure shown in Figure 5 becomes an inhomogeneous composite system having two regions with different thermal conductivities. We assume that the two regions have same thermal conductivity coefficient $\kappa$ along $x$-direction and different $\kappa_{1}$ and $\kappa_{2}$ along $z$-direction having an imperfect interface at $z=h_{1}+L, x=(0, \delta)$. Some boundaries of this system are thermally insulated. In actual modeling, thermal conditions could be more intense, which lead to some different rigid boundary conditions, but they can always be incorporated into the scheme. We aim to suggest a robust and efficient approach to simulate steady state heat conduction in a composite region, described in Figure 5. Hence, based on the made assumptions, we require to solve the following elliptic equation to determine temperature distribution in system shown in Figure 5:

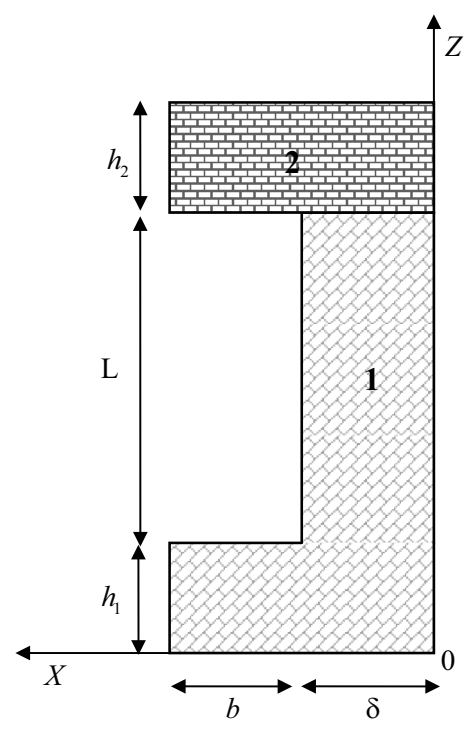

Fig. 5. A recurring element of the structure of problem 2
In region 1.

$$
\frac{\partial}{\partial x}\left(\kappa \frac{\partial U_{1}}{\partial x}\right)+\frac{\partial}{\partial z}\left(\kappa_{1} \frac{\partial U_{1}}{\partial z}\right)=0
$$

with boundary conditions

$$
\begin{aligned}
& \frac{\partial U_{1}(0, z)}{\partial x}=0, \quad \forall z \in\left[0, h_{1}+L\right], \\
& \frac{\partial U_{1}(\delta, z)}{\partial x}=0, \quad \forall z \in\left[h_{1}, h_{1}+L\right], \\
& U_{1}(x, 0)=U_{0}, \quad \forall x \in[0, b+\delta], \\
& \frac{\partial U_{1}(b+\delta, z)}{\partial x}=0, \quad \forall z \in\left[0, h_{1}+L\right], \\
& U_{1}\left(x, h_{1}\right)=U_{K}, \quad \forall x \in[\delta, b+\delta] .
\end{aligned}
$$

In region 2.

$$
\frac{\partial}{\partial x}\left(\kappa \frac{\partial U_{2}}{\partial x}\right)+\frac{\partial}{\partial z}\left(\kappa_{2} \frac{\partial U_{2}}{\partial z}\right)=0 ;
$$

with boundary condition

$$
\begin{aligned}
& U_{2}\left(x, h_{1}+L\right)=U_{L}, \forall x \in[\delta, b+\delta], \\
& \frac{\partial U_{2}(0, z)}{\partial x}=0, \forall z \in\left[h_{1}+L, h_{1}+L+h_{2}\right], \\
& \frac{\partial U_{2}(b+\delta, z)}{\partial x}=0, \forall z \in\left[h_{1}, h_{1}+L\right], \\
& U_{2}\left(x, h_{1}+L+h_{2}\right)=U_{M}, \forall x \in\left[0, h_{1}+L+h_{2}\right] .
\end{aligned}
$$

Contact conditions between region 1 and region 2 .

$$
\left.\begin{array}{c}
U_{2}\left(x, h_{1}+L\right)=U_{1}\left(x, h_{1}+L\right)+J_{1}, \\
\frac{\partial U_{2}\left(x, h_{1}+L\right)}{\partial z}=\frac{\partial U_{1}\left(x, h_{1}+L\right)}{\partial z}+J_{2}, \\
\kappa_{2} \frac{\partial U_{2}\left(x, h_{1}+L\right)}{\partial z}=\kappa_{1} \frac{\partial U_{1}\left(x, h_{1}+L\right)}{\partial z}+J_{3}
\end{array}\right\} \forall x \in(0, \delta) .
$$

The values of parameters that we used in the computation are:

$\kappa=100 \mathrm{~W} / \mathrm{m} . \mathrm{K}, \kappa_{1}=290 \mathrm{~W} / \mathrm{m} \cdot \mathrm{K}, \kappa_{2}=14.5 \mathrm{~W} / \mathrm{m} . \mathrm{K}$, $\delta=0.001 \mathrm{~m}, b=0.001 \mathrm{~m}, h_{1}=0.001 \mathrm{~m}, L=0.002 \mathrm{~m}$, $h_{2}=0.003 \mathrm{~m}, U_{0}=25 \mathrm{~K}, U_{K}=40 \mathrm{~K}, U_{L}=75 \mathrm{~K}$, $U_{M}=150 \mathrm{~K}, J_{1}=25 \mathrm{~K}, J_{2}=20000 \mathrm{~K} / \mathrm{m}$, $J_{3}=-1087500 \mathrm{~K} / \mathrm{m}^{2}$.

We have plotted temperature distribution at line $x=0.0005 \mathrm{~m}$ in $12 \times 12$ grid in Figure 6 to visualize the variation of temperature along height of the composite system. It can be easily seen that the temperature rises along the height. Descretization of such an inhomogeneous domain along with insulated boundaries and discontinuous contact conditions becomes very difficult. Any arbitrary set of grids may not be well suited for this domain; hence we have used number 


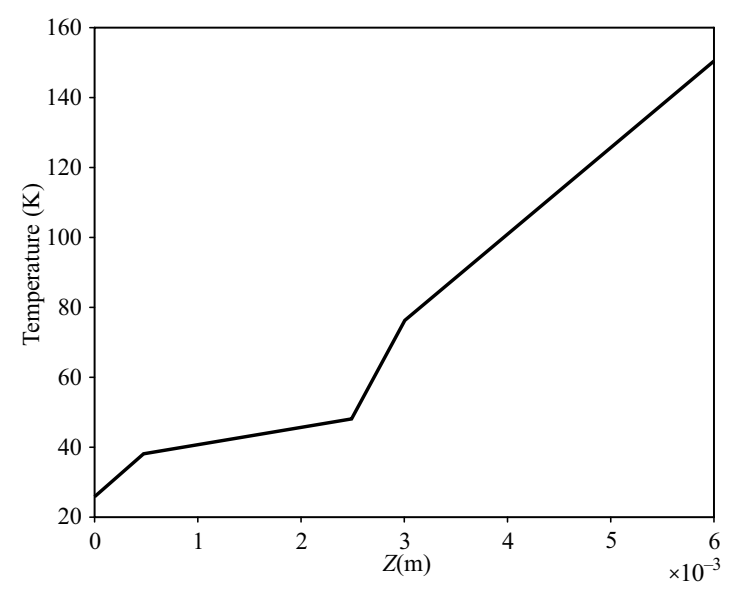

Fig. 6. Temperature profile of composite system in problem 2 at cross section $x=0.0005 \mathrm{~m}$ in $12 \times 12$ grid

of subintervals in the multiple of 6 so that the grids fit in the domain properly. Table 2 and Table 3 display temperature of the system at some selected points in region 1 and region 2 respectively in $12 \times 12$ grid.

Table 2. Temperature at different arbitrary points in region 1

\begin{tabular}{|c|c|}
\hline $\begin{array}{c}\text { Rectangular Co-ordinates }(\mathrm{m}) \\
(x, z)\end{array}$ & Temperature $(\mathrm{K})$ \\
\hline$(0.0008333,0.0005)$ & 37.50 \\
\hline$(0.0003333,0.002)$ & 45.00 \\
\hline$(0.0006667,0.0025)$ & 47.50 \\
\hline
\end{tabular}

Table 3. Temperature at different arbitrary points in region 2

\begin{tabular}{|c|c|}
\hline $\begin{array}{c}\text { Rectangular Co-ordinates }(\mathrm{m}) \\
(x, z)\end{array}$ & Temperature $(\mathrm{K})$ \\
\hline$(0.0015,0.0035)$ & 87.50 \\
\hline$(0.0003333,0.004)$ & 100.00 \\
\hline$(0.0001167,0.0055)$ & 137.50 \\
\hline
\end{tabular}

\section{Conclusions}

We have determined steady state temperature distribution using decomposed immersed interface method in two inhomogeneous composite systems having two regions with imperfect interface i.e. there exist some discontinuities along the interface. In first problem, we have also proved the effectiveness and accuracy of the approach by comparing it with the analytical solution. Since very few methods in the existing literature can handle a steady state heat conduction problem in a composite system with discontinuities in temperature and its derivatives, this approach may be very beneficial to deal a variety of industrial problems and engineering applications.

\section{References}

Ang, W.-T. 2006. Non-steady state heat conduction across an imperfect interface: a dual-reciprocity boundary element approach, Engineering Analysis with Boundary Elements 30: 781-789.

http://dx.doi.org/10.1016/j.enganabound.2006.03.005

Ang, W.-T.; Choo, K. K.; Fan, H. 2004. A Green's function for steady-state two-dimensional isotropic heat conduction across a homogeneously imperfect interface, Communications in Numerical Methods in Engineering 20: 391-399. http://dx.doi.org/10.1002/cnm.681

Balabukh, L. I.; Kolesnikov, K. S.; Zarubin, V. S., et al. 1969. Osnovy stroitel'noi mekhaniki raket [Fundamentals of Construction mechanics of Rockets]. Vysshaya Shkola, Moscow.

Berger, J. R.; Skilowitz, J. L.; Tewary, V. K. 2000. Green's function for steady-state heat conduction in a bimaterial composite solid, Computational Mechanics 25(6): 627-633.

http://dx.doi.org/10.1007/s004660050509

Berthelsen, P. A. 2004. A decomposed immersed interface method for variable coefficient elliptic equations with nonsmooth and discontinuous solutions, Journal of Computational Physics 197(1): 364-386.

http://dx.doi.org/10.1016/j.jcp.2003.12.003

Datskovskii, V. A.; Yakunin, A. N. 2005. Steady-state heat conduction in composite systems with boundary conditions of the fourth kind, Journal of Engineering Physics and Thermophysics 36(1): 107-110. http://dx.doi.org/10.1007/BF00861301

Haji-Sheikh, A.; Beck, J. V.; Agonafer, D. 2003. Steady-state heat conduction in multi-layer bodies, International Journal of Heat and Mass Transfer 46(13): 2363-2379. http://dx.doi.org/10.1016/S0017-9310(02)00542-2

Joshi, P.; Kumar, M. 2012. Mathematical model and computer simulation of three dimensional thin film elliptic interface problems, Computers \& Mathematics with Applications 63(1): 25-35. http://dx.doi.org/10.1016/j.camwa.2011.10.054

Kumar, M.; Joshi, P. 2012a. A mathematical model and numerical solution of a one dimensional steady state heat conduction problem by using high order immersed interface method on non-uniform mesh, International Journal of Nonlinear Science 14(1): 11-22.

Kumar, M.; Joshi, P. 2012b. Some numerical techniques to solve elliptic interface problems, Numerical Methods for Partial Differential Equations 28(1): 94-114.

http://dx.doi.org/10.1002/num.20609

Kumar, M.; Joshi, P. 2013. Mathematical modelling and computer simulation of steady state heat conduction in anisotropic multi-layered bodies, International Journal of Computing Science and Mathematics 4(2): 85-119.

http://dx.doi.org/10.1504/IJCSM.2013.055195

Li, Z. 1994. The immersed interface method - a numerical approach for partial differential equations with interfaces: $\mathrm{PhD}$ thesis. University of Washington.

Ma, C.-C.; Chang, S.-W. 2004. Analytical exact solutions of heat conduction problems for anisotropic multi-layered media, International Journal of Heat and Mass Transfer 47(8-9): 1643-1655.

http://dx.doi.org/10.1016/j.ijheatmasstransfer.2003.10.022 
Noor, A. K.; Burton, W. S. 1991. Steady state heat conduction in multilayered composite plates and shells, Computers and Structures 39: 185-193.

http://dx.doi.org/10.1016/0045-7949(91)90086-2

Seyidmamedov, Z. M.; Ozbilge, E. 2006. A mathematical model and numerical solution of interface problems for steady state heat conduction, Mathematical Problems in Engineering,1-18. http://dx.doi.org/10.1155/MPE/2006/20898
Xia, Y.; Jacobi, A. M. 2004. An exact solution to steady state heat conduction in a two-dimensional slab on a one-dimensional fin: application to froasted heat exchangers, International Journal of Heat and Mass Transfer 47(14-16): 3317-3326. http://dx.doi.org/10.1016/j.ijheatmasstransfer.2004.01.019

Zarubin, V. S.; Radikov, A. V. 2007. Mathematical simulation of the temperature state of an inhomogeneous body, High Temperature 45(2): 243-254. http://dx.doi.org/10.1134/S0018151X07020150

Pratibha JOSHI. Dr. Pratibha Joshi is assistant professor in department of mathematics at University of Petroleum \& Energy Studies, Dehradun (Utarakhand), India. She has been working on the area of numerical techniques and computational mathematics for past seven years. She has completed her post graduation degree in applied mathematics from IIT Roorkee and her PhD degree from NIT Allahabad. She has also published many research papers in international journals.

Manoj KUMAR. Dr Manoj Kumar obtained his PhD degree in 2000 from Aligarh Muslim University Aligarh India in Applied Mathematics and presently working as Associate Professor department of Mathematics, Motilal Nehru National Institute of Technology, Allahabad-211004 (U.P) India. He has guided ten PhD students and published more than sixty research papers in international journals. 